\title{
Raman induced phase conjugation spectroscopy
}

\author{
B RAGHAVENDRA PRASAD, R KRISHNA MOHAN, \\ C K SUBRAMANIAN and P S NARAYANAN* \\ Department of Physics, Indian Institute of Science, Bangalore 560012, India
}

\begin{abstract}
Raman induced phase conjugation (RIPC) spectroscopy is a relatively new coherent Raman spectroscopic (CRS) technique using optical phase conjugation (OPC), with which complete Raman spectra of transparent media can be obtained. It is a non-degenerate four-wave mixing technique in which two pulsed laser beams at $\omega_{l}$ and $\omega_{l} \pm \Delta$; where $\Delta$ corresponds to a vibrational frequency of a nonlinear medium $\}$ mix with a third laser beam at $\omega_{1}$ to generate a fourth beam $\omega_{1} \pm \Delta$, which is nearly phase conjugate to one of the beams at $\omega_{I}$. With this technique one can measure the ratio of the resonant and nonresonant components of the third-order nonlinear susceptibilities of the nonlinear media. We have used this technique to get Raman spectra of well-known organic solvents like benzene etc., using pulsed Nd: YAG - dye laser systems. We have also studied the effect of delaying one of the interacting beams with respect to the others and the phase conjugate property of RIPC signals.
\end{abstract}

Keywords. Raman induced phase conjugation spectroscopy; coherent Raman spectroscopy; phase conjugate property.

\section{Introduction}

Optical Phase Conjugation (OPC) has been obtained by the use of several nonlinear optical effects, to reverse exactly both the direction of propagation and the overall phase factor of a coherent beam of light. This wavefront or phase reversal of an electromagnetic wave is equivalent to performing an operation of complex conjugation of the complex spatial amplitude. This phenomenon has been observed with all states of matter using various nonlinear optical effects. OPC has many wide-ranging applications both in the theoretical and practical fields. Some of the potential applications of OPC include real time adaptive optics (compensation of aberrated fields due to propagation on image transmission through distorting media), optical signal processing (in the time domain and on the spatial domain), image processing, optical computing, ultra slow noise detection, interferometry and nonlinear laser spectroscopy (Pepper 1982; Fischer 1983; Zel'dovich et al 1985).

Several Coherent Raman Spectroscopic (CRS) techniques which can give resolution of greater than $1 \mathrm{~cm}^{-1}$, are being used to obtain the optical spectra of different media even in the presence of strong fluorescence, and at high temperatures when a strong thermal background is present etc. Most coherent Raman spectroscopic techniques such as CARS, RIKES, SRS etc use two different lasers, whose frequency difference

\footnotetext{
* For correspondence
} 
can be tuned so as to match one of the Raman excitation frequencies of the sample under study. Recently a new CRS technique has been proposed and demonstrated by Saha and Hellwarth (1983). This technique makes use of OPC by a nondegenerate four-wave mixing (NFWM) technique to obtain a Raman spectrum of the sample. This new spectroscopic technique, because of its phase conjugation principle, has been christened Raman Induced Phase Conjugation (RIPC) spectroscopy. Here two single-pulse laser beams at $\omega_{l}$ and $\omega_{l} \pm \Delta$ (where $\Delta$ corresponds to vibrational frequency of the nonlinear medium) mix with a third laser beam at $\omega_{l}$ to generate a fourth beam at $\omega_{t} \pm \Delta$, which is nearly phase conjugate to one of the beams at $\omega_{t}$. The Raman signal frequencies obtained in RIPC are equal to $\omega_{4}=\omega_{1}+\omega_{2}-\omega_{3}$ where $\omega_{1}=\omega_{2}=\omega_{l}, \omega_{4}=\omega_{3}=\omega_{s}=\omega_{l} \pm \Delta$. The resonance enhancement of the $\chi^{(3)}$ generates the signals at the Stokes or anti-Stokes frequencies.

The main characteristics of this technique are (a) wide frequency range (thousands of $\mathrm{cm}^{-1}$ ) and a broad acceptance angle for phase matching among the interacting beams thus allowing an enhanced phase conjugate signal at Raman resonance, (b) 16 independent combinations of beam polarizations are possible, and if the beam polarizations are adjusted properly, only Raman-shifted frequencies are conjugated, (c) the Raman signal is nearly phase conjugate to one of the interacting beams and is not coincident with any of the incident beams thus giving an enhanced spatial resolution. In this paper we have used the RIPC technique to obtain Raman spectra of some organic solvents such as benzene, nitrobenzene, toluene and acetone in the frequency shift range 750 to $1400 \mathrm{~cm}^{-1}$ to examine the relative intensities of different Raman lines. The effects of introducing time delays in one of the interacting beams with respect to the others and the phase conjugation property of the RIPC signal have also been demonstrated.

\section{Theory}

RIPC is a form of coherent, nondegenerate four-wave mixing (NFWM) technique and the process is illustrated in figure 1. Here a Raman vibration(s) of the medium is excited by the simultaneous presence of the forward propagating probe beam (2) at $\omega_{l}$ and the backward propagating broad band read beam (3) at frequency $\omega_{l} \pm \Delta$ excites the Raman modes of the sample. The forward propagating pump beam (1) at $\omega_{l}$ sees the variation of polarizability due to this excitation and consequently a portion of it gets scattered as the RIPC signal (Saha and Hellwarth 1983; McCallum and Hutchinson 1987; McCallum et al 1988) at a frequency $\omega_{l} \pm \Delta$, whose phase front is nearly phase conjugate to that of probe beam (2). This interaction occurs because of third order nonlinear susceptibility and the interacting electric fields are assumed to be monochromatic electric fields with slowly varying complex amplitude represented by

$$
E_{n}\left(\omega_{n}, z, t\right)=\frac{1}{2}\left\{E_{n}\left(\omega_{n}, z\right) \exp \left[-i\left(\omega_{n} t-k_{n} z\right)\right]+c \cdot c\right\} .
$$

In order to understand and calculate the spectrum obtained in RIPC it is necessary to define $\chi^{(3)}$ in terms of coefficients defined by Hellwarth (1977). Even though $\chi^{(3)}$ is a fourth rank tensor containing 81 elements, only four of them are relevant to 


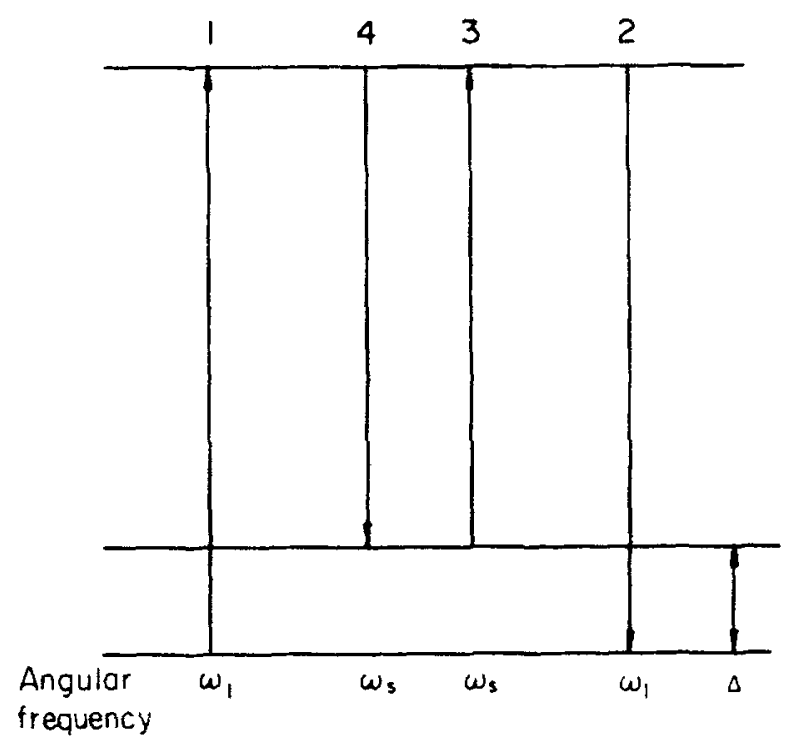

Figure 1. Energy level diagram for RIPC.

RIPC in an isotropic medium. They are

$$
\begin{aligned}
& \chi_{1111}=3 \sigma+2\left(A_{0}+B_{0}+A_{\mu}+B_{\mu}\right), \\
& \chi_{1122}=\sigma+2 A_{0}+B_{\mu}, \\
& \chi_{1221}=\sigma+B_{0}+B_{\mu}, \\
& \chi_{1212}=\sigma+B_{0}+2 A_{\mu} .
\end{aligned}
$$

Here $\sigma$ is a number measuring the instantaneous nonlinear electronic response or hyperpolarizability, $A_{\mu}$ and $B_{\mu}$ are the nuclear response functions describing Raman active excitations which alter the optical susceptibility of the medium in which four-wave mixing takes place, $A_{0}$ and $B_{0}$ are defined at line centre (i.e. zero Raman shift) and both are entirely real. Here $A_{0}, B_{0}$ and $\sigma$ vary only slightly with frequency as long as the Born-Oppenheimer approximation is valid, i.e., when all optical frequencies are well below the electronic band edge. Thus we have

$$
\operatorname{Im} B_{\Delta}=\left[\frac{8 \pi^{3} n_{l} \varepsilon_{0}^{2} c^{4}}{3 n_{s} h \omega_{l} \omega_{s}^{3}}\right]\left[\frac{\mathrm{d}^{2} \sigma_{\perp}}{\mathrm{d} \Omega \mathrm{d} \omega_{s}}\left(1-\overrightarrow{\boldsymbol{e}}^{\gamma}\right)\right]
$$

where $d^{2} \sigma_{1} / d \Omega d \omega_{s}$ is the peak Raman scattering cross-section which gives the probability per unit length of a photon scattering at frequency $\omega_{l}$ to frequency $\omega_{s}$ per unit angular frequency range per unit solid angle for perpendicular polarization (units $\mathrm{m}^{-1} \mathrm{sr}^{-1} \mathrm{~s} \mathrm{rad}^{-1}$ ), $\Omega, \varepsilon_{0}, c$ and $h$ are solid angle of emission, permittivity, speed of light and Planck's constant respectively, $\Delta=\omega_{l}-\omega_{s}, y=\left[\hbar\left(\omega_{l}-\omega_{s}\right)\right] k T$ where $k$ is the Boltzman's constant, $T$ the temperature, $n_{l}$ the refractive index at $\omega_{l}$ and $n_{s}$ the 
refractive index at $\omega_{s}$. Similarly we have

$$
\operatorname{Im} A_{\Delta}=\left[\frac{8 \pi^{3} n_{l} \varepsilon_{0}^{2} c^{4}}{3 n_{s} \hbar \omega_{l} \omega_{s}^{3}}\right]\left[\frac{1}{2}\left(\frac{\mathrm{d}^{2} \sigma_{\|}}{\mathrm{d} \Omega \mathrm{d} \omega_{s}}-\frac{\mathrm{d}^{2} \sigma_{\perp}}{\mathrm{d} \Omega \mathrm{d} \omega_{s}}\right)\right]\left(1-e^{-\gamma}\right),
$$

where $\mathrm{d}^{2} \sigma_{1} / \mathrm{d} \Omega \mathrm{d} \omega_{s}$ is the peak differential Raman scattering cross-section per unit volume for parallel polarization. Because of the causal nature of $A_{\mu}$ and $B_{\mu}$ the real parts of $A_{\Delta}$ and $B_{\Delta}$ can be calculated from the imaginary parts, (3) and (4), by the Kramers- Kronig integrals. Given the Raman spectrum of a medium and the electronic hyperpolarizability, the nonlinear polarization density can be completely determined. From (3) and (4), the ratio between $A_{\mu}$ and $B_{\mu}$, for a Raman line with depolarization ratio $\rho=\sigma_{\perp} / \sigma_{i}$, is given by

$$
\frac{B_{\mu}}{A_{\mu}}=\frac{2 \rho}{(1-2 \rho)} .
$$

$A_{\mu}$ and $B_{\mu}$ both have real and imaginary parts and are significant only in the region of Raman resonances. Hence $A_{\mu}$ and $B_{\mu}$ are responsible for lines in RIPC spectra.

In the experiments $\bar{E}_{n}$ is.essentially the sum of three incident beams $\bar{E}_{1}+\bar{E}_{2}+\bar{E}_{3}$ which give rise to a term in the polarization density which generates a fourth-phase conjugate beam at $\bar{E}_{4}$. By taking only $\left[\operatorname{Re} \bar{P}_{4} \exp i\left(\bar{k}_{4} \cdot \bar{x}-\omega_{s} t\right)\right]$, the effective nonlinear polarization component that gives rise to the phase conjugation $\left(A_{\mu}=A_{-\Delta}=A_{\Delta}^{*}\right.$, $\left.A_{0}=A_{\Delta}=A_{0}^{*}\right)$ is given by

$$
\begin{aligned}
\bar{P}_{4}=\frac{1}{4}\left[\overline { E } _ { 1 } \overline { E } _ { 2 } ^ { * } \overline { E } _ { 3 } \left(\sigma+B_{0}+\right.\right. & \left.2 A_{\mu}\right)+\bar{E}_{2}^{*} \bar{E}_{1} \bar{E}_{3}\left(\sigma+B_{0}+B_{\mu}\right)+ \\
& \left.+\bar{E}_{3} \bar{E}_{2}^{*} \bar{E}_{1}\left(\sigma+2 A_{0}+B_{\mu}\right)\right] \exp [i \Delta \bar{k} \cdot \bar{x}] .
\end{aligned}
$$

For different polarization combinations of interacting beams, the appropriate polarization component is chosen and the precise spectral shape depends on this combination.

To optimize the RIPC signal it is necessary to phase match the beams. The signals fall to half maximum whenever the magnitude $\Delta k$ of the beam wave vector mismatch $\Delta \bar{k}=\bar{k}_{1}-\bar{k}_{2}+\bar{k}_{3}-\bar{k}_{4}$ exceeds either the absorption coefficient $\alpha$ (where $\alpha L>1$ ) or $2 \cdot 8 / L$ (when $\alpha L<1$ ). When the angle of the probe beam (angle of interaction) is varied by $\Delta \theta$ from $\theta$, the resulting $\Delta k$ is

$$
\Delta k=\left|\frac{1}{2} k_{2}\left[\left(k_{2} / k_{4}\right)-1\right] \theta \Delta \theta\right|,
$$

if $\theta$ is kept fixed and only the frequency of the read beam is varied by $\Delta v$, then the resulting phase mismatch $\Delta k$ is

$$
\Delta k=\left|\left[\theta_{1}^{2}-1+\left(k_{4} / k_{3}\right)\right] n_{3}(\Delta / c)\right|
$$

where $\theta_{1}$ is the angle between the probe beam and the signal beam. From (7) and (8) the process is still phase-matched adequately (i.e., the efficiency of the process is more than half the full efficiency obtained when there is a perfect phase matching i.e., $\Delta k=0$ ) even when $\theta$ is varied by \pm 20 milliradians or the frequency detuning of the read beam is about $\pm 200 \mathrm{~cm}^{-1}$. These ranges are one to two orders of magnitude larger than that available in CARS. Therefore a broad band read beam can be used to generate the RIPC signal. 
If the incident beams are negligibly disturbed by the mixing process, the intensity $I_{4}$ of the phase conjugate beam can be calculated from (6) in terms of incident beam intensities $I_{1}, I_{2}$ and $I_{3}$. The result is written as

$$
I_{4} / I_{3}=\beta^{2} L^{2} I_{1} I_{2}
$$

where $\beta$ is the nonlinear coefficient which depends on beam polarizations and the other parameters in (6) and $L$ is the interaction length.

\section{Experiments and results}

The experimental investigations of RIPC were made using the arrangement shown in figure 2. The primary laser is a Quanta Ray (DCR-2A) Nd:YAG laser with filledin beam configuration. The second harmonic of this laser was used to pump a Quanta Ray (PDL-II) dye laser. The dye laser could be used either as a broad band source or as a narrow band source with tuning elements. The output of the dye laser is from $550-580 \mathrm{~nm}$ with Rh6G-methanol as active medium. The pulse widths of the laser beams are 6-8ns. The output of the Nd:YAG laser was split into two parts by the beam splitter $\mathbf{B S}_{1}$. One beam is used to pump the dye laser and the second beam is further split into two parts by $\mathrm{BS}_{2}$. The reflected beam is known as the probe beam and the transmitted one as the pump beam. The output of the dye laser is used as the read beam. The forward propagating pump beam and the backward propagating read beam were made collinear and counterpropagating using the mirrors $\mathbf{M}_{3}$ and

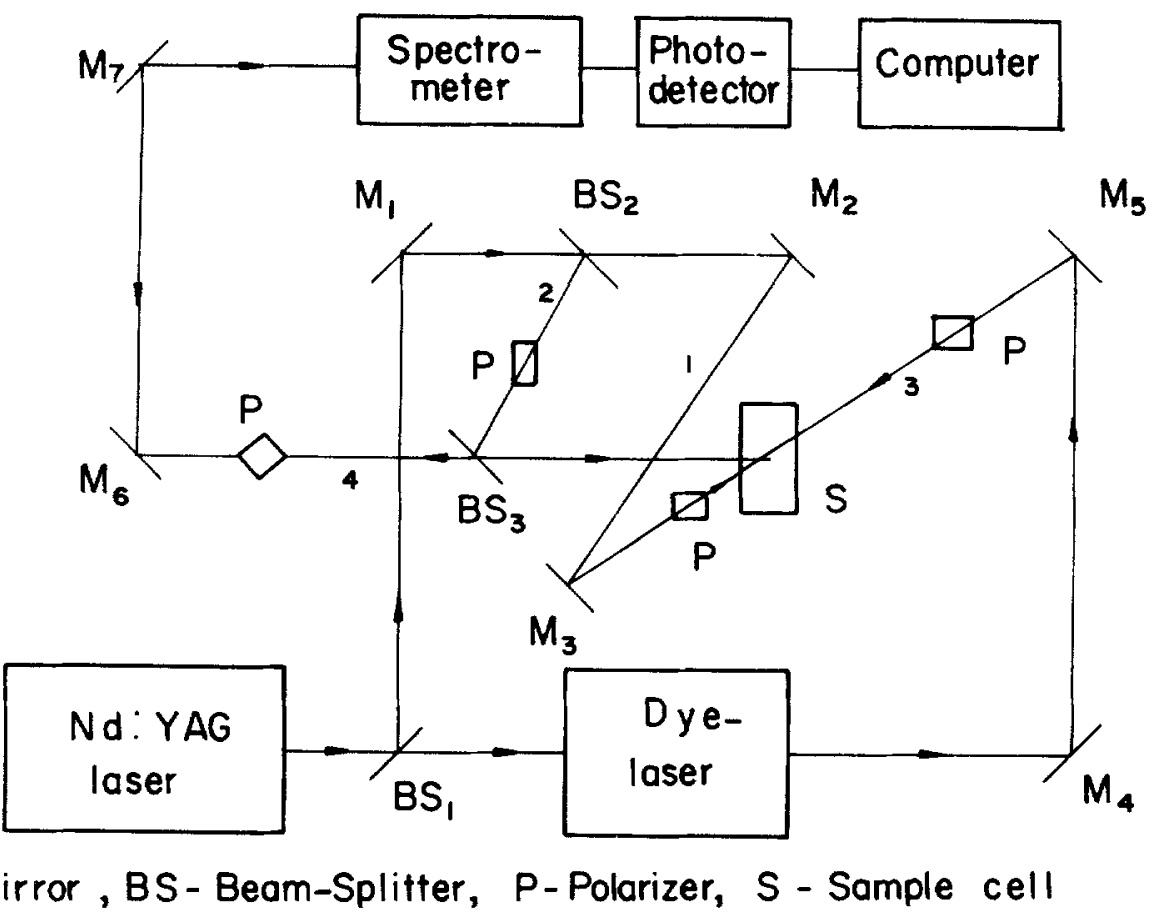

Figure 2. Experimental layout for RIPC studies. 
$M_{5}$. It was found that to generate RIPC signals it is an absolute must that all the interacting beams arrive at the same time in the sample cell. This is achieved by introducing necessary delays in the appropriate beams. The RIPC signal is passed through a spectrometer (to ensure that spurious scattered light does not fall on the detector) which is connected to the dye laser for synchronised scanning and is detected by a photodetector placed at the output of the spectrometer. The detected RIPC signals are transferred to a computer for storage and subsequent analysis.

The experiment was carried out by fixing the wavelengths of the probe and pump beams and scanning the read beam by a motor-controlled interface connected to a computer. The samples studied are double distilled analar grade benzene, nitrobenzene, acetone and toluene. For every $0 \cdot 1 \mathrm{~nm}$ scan of the read beam the RIPC signal was averaged over 100 pulses and transferred to an $\mathrm{HP}-9836 \mathrm{C}$ computer for analysis and plotting. The RIPC spectrum thus obtained for four samples using perpendicularly polarized interacting beams are shown in figures 3-6. All the prominent Raman lines of the samples in the region 750 to $1400 \mathrm{~cm}^{-1}$ can be seen in the figures. The relative intensities of different lines are found to be in agreement with those of the lines taken using conventional Raman spectroscopic techniques (Schrader and Meier 1974). For time-resolved measurements, the pump or the probe beams can be delayed relative to the other two beams and the intensity of the RIPC signal could be measured for different delays. By introducing delay in the probe beam one can measure either the coherence or the intensity correlation of the exciting beam. The dependence of the RIPC intensity (as measured for the $992 \mathrm{~cm}^{-1}$, i.e. the totally symmetric mode of benzene) is shown in figure 7. The signal intensity falls sharply on either side of zero time delay. This also shows that to generate RIPC signals, the simultaneous presence of interacting beams is necessary. Using RIPC the vibrational relaxation and phonon

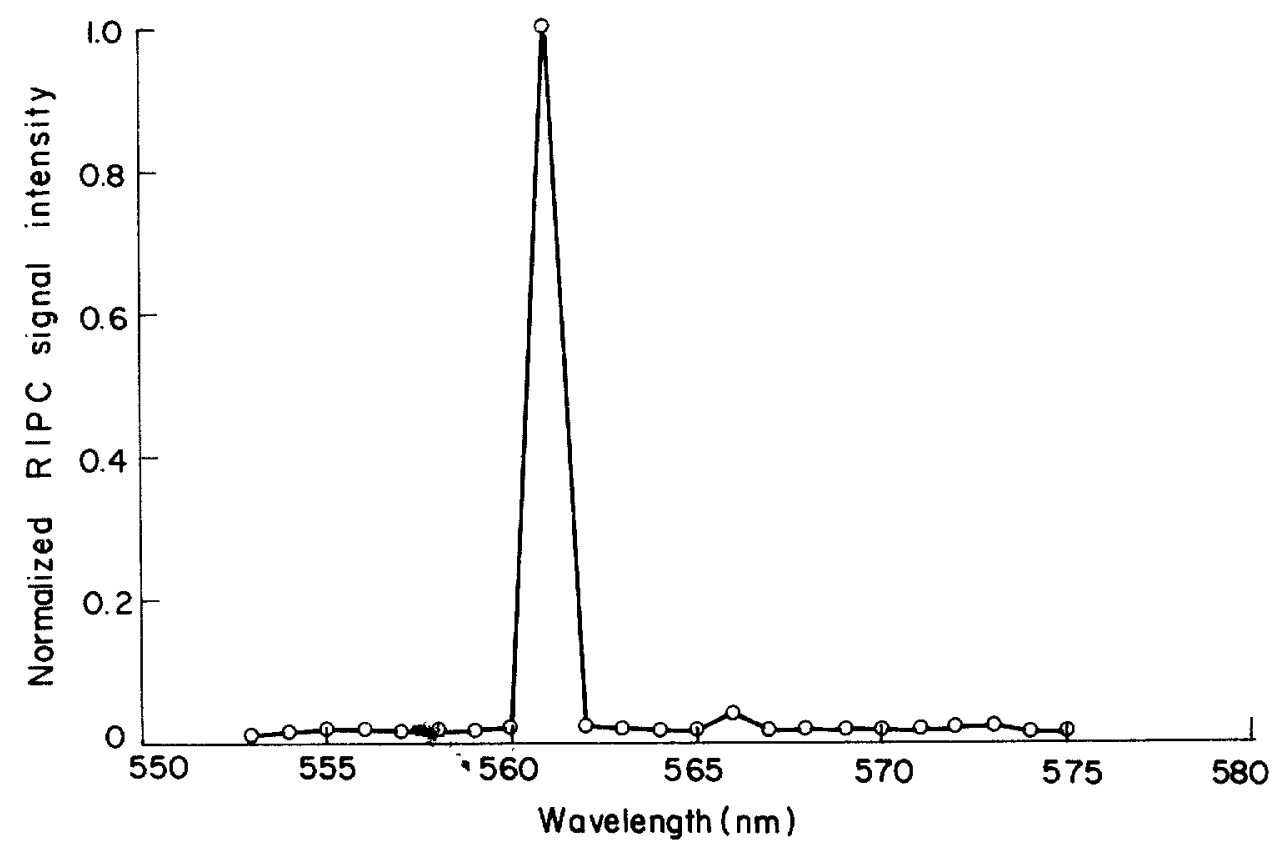

Figure 3. RIPC spectrum of benzene. 


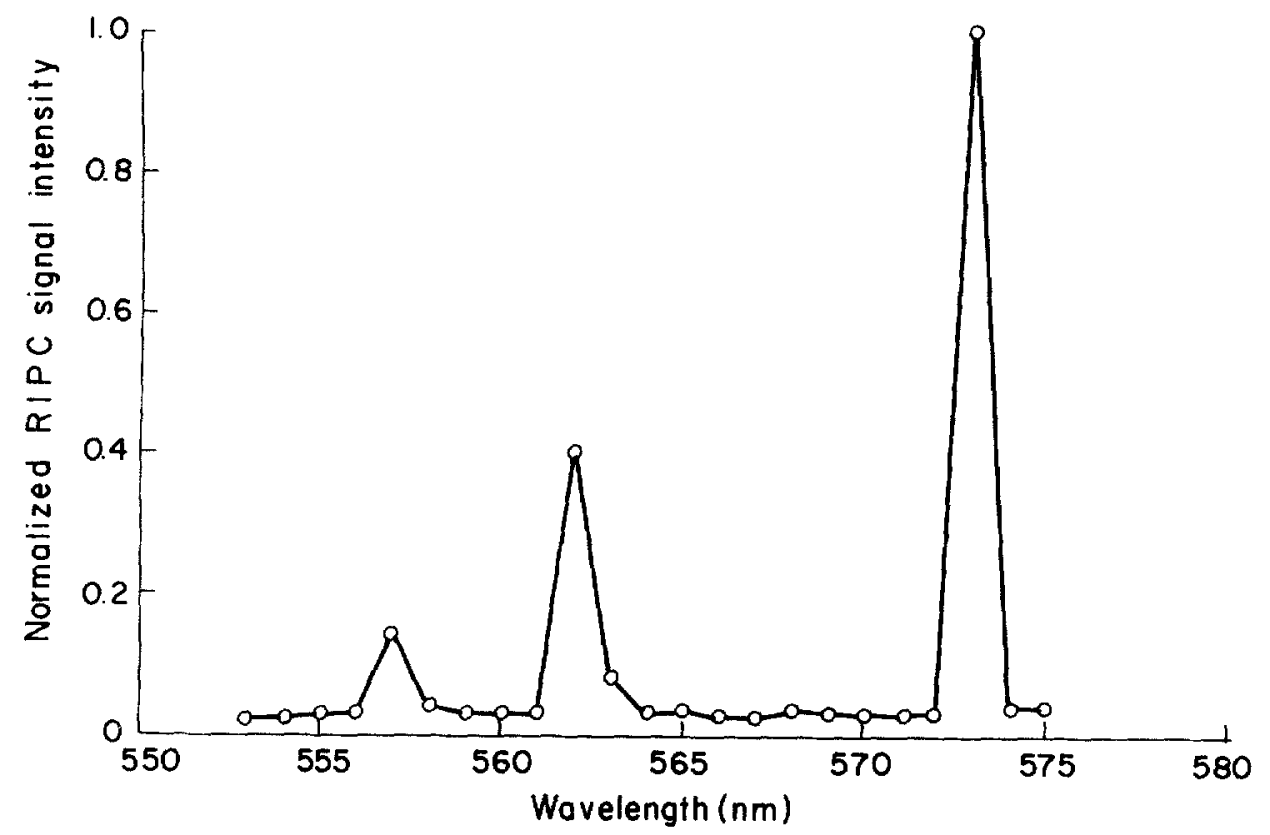

Figure 4. RIPC spectrum of nitrobenzene.

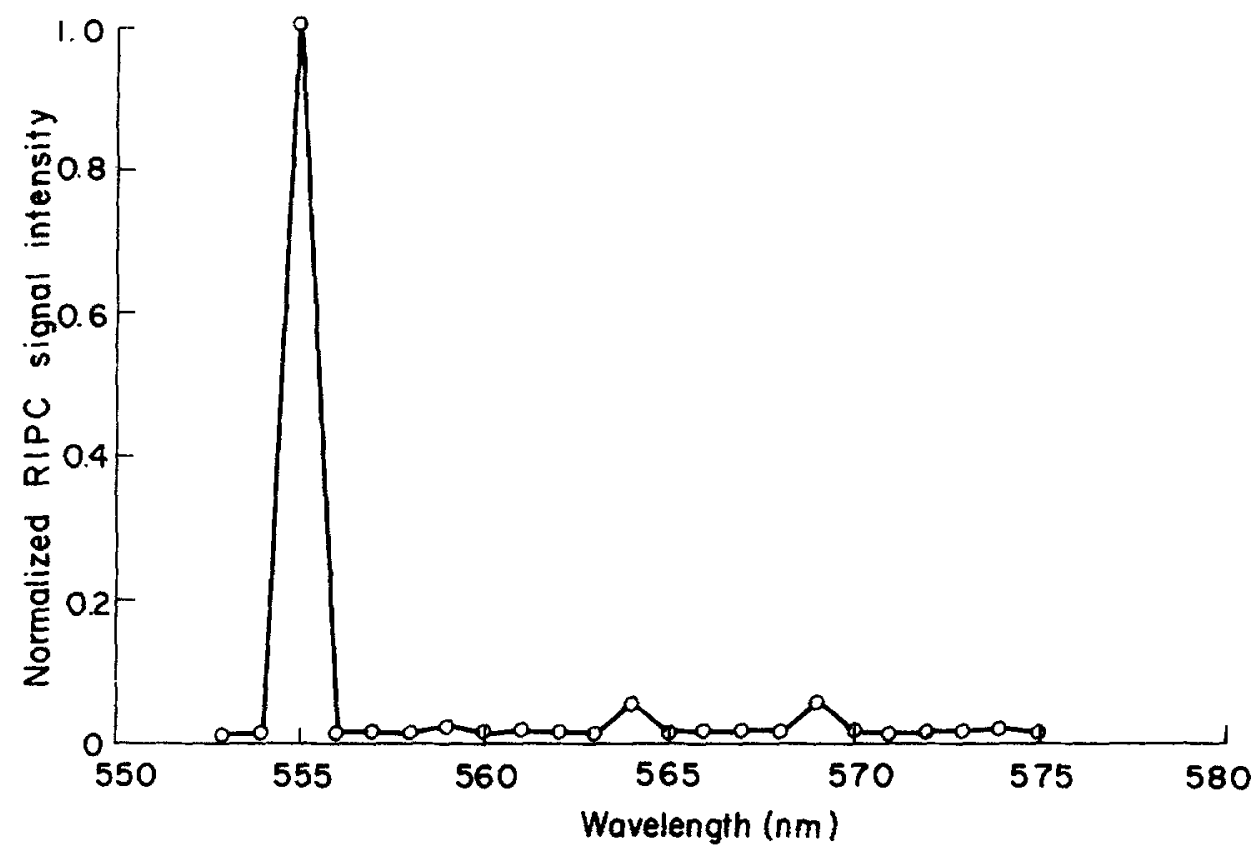

Figure 5. RIPC spectrum of acetone. 


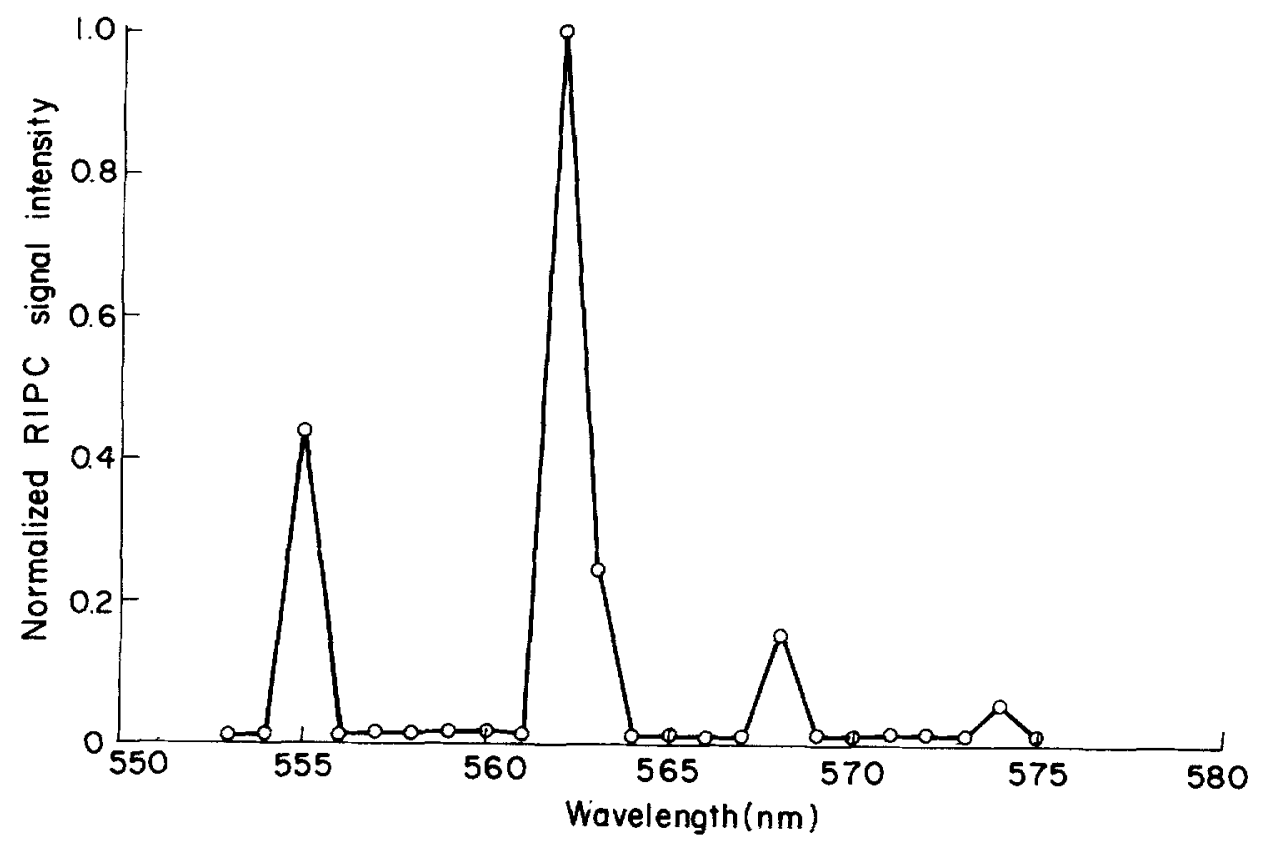

Figure 6. RIPC spectrum of toluene.

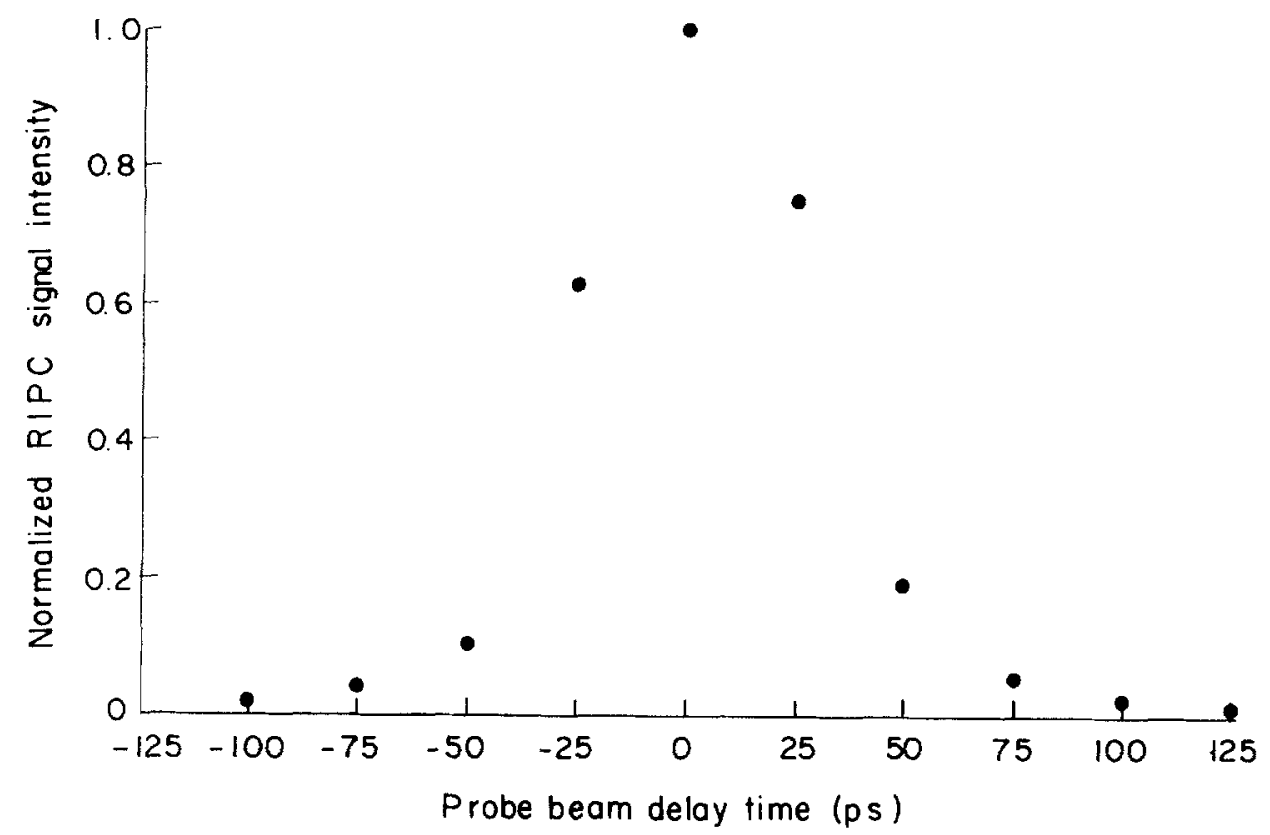

Figure 7. Dependence of the RIPC signal of the $992 \mathrm{~cm}^{-1}$ totally symmetric mode of benzene on the probe beam delay. 

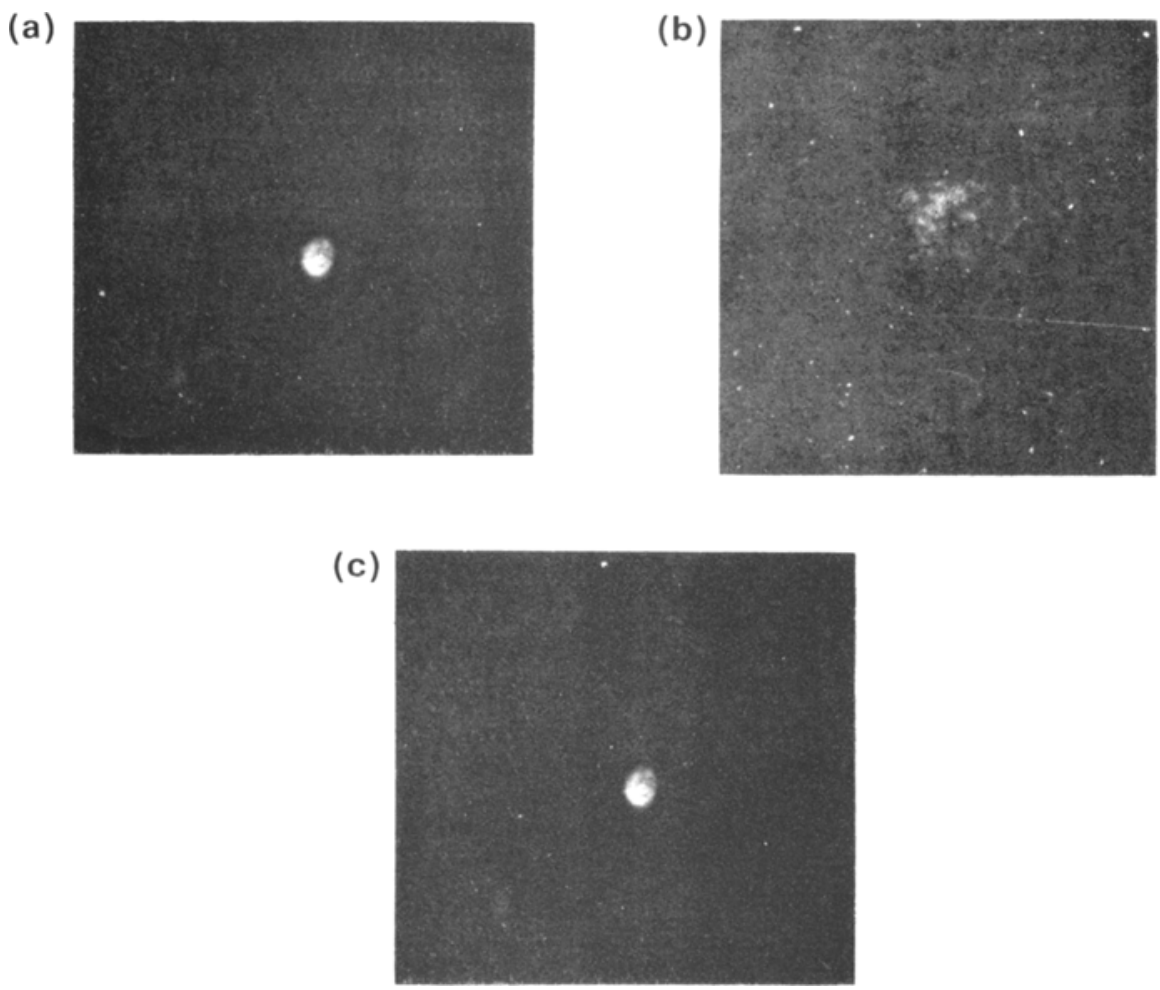

Figure 8. Phase conjugation property of RIPC signal: (a) Original RIPC signal without the distorter in the path of the probe beam. (b) Distorted probe beam. (c) Phase conjugate beam (RIPC signal) after the reverse passage through the distorter.

dephasing times have been measured with picosecond resolutions, by Dorsinville et al (1987) and Delfyett et al (1987).

The phase conjugation properties of the Raman signals are shown in figure $8 \mathrm{a}-\mathrm{c}$. Figure 8 a shows the beam shape of the RIPC signal without any phase distorter in the path of the probe beam. Figure $8 \mathrm{~b}$ shows the distorted probe beam when a phase distorter is placed in its path. When the RIPC signal is generated, on its reverse passage through the phase distorter, because of its phase reversal nature, all the distortions are removed. These pictures conclusively prove that the Raman signals obtained by this technique are phase conjugated.

\section{Conclusions}

The advantages of RIPC as a spectroscopic tool over other CRS techniques are many. The Raman spectra of any transparent media can be obtained through this technique. Raman spectra of samples with unknown refractive indices can be obtained easily through RIPC due to its broad phase matching angles as compared to other CRS methods where a tedious search for phase matching angle is required. Time-resolved RIPC provides a direct measurement of the relaxation times of the excited vibrational modes and the phonon dephasing times of both liquids and solids (Delfyett et al 1987; 
Dorsinville et al 1987). The resolution of RIPC techniques depends on the line widths of the laser beams. Another important advantage of RIPC is that the Raman spectrum of a system can be got even when: (a) it is undergoing phase transitions at very high temperatures, (b) undergoing continuous temperature fluctuations, and (c) the temperature is very high and it is difficult to obtain the spontaneous Raman spectrum because of predominant thermal background. Since RIPC can record the entire Raman spectrum in a single shot, this technique can be used in combustion diagnostics and in detection of different species. Thus RIPC is a very powerful tool for obtaining the Raman spectra of any system as compared to other CRS techniques.

\section{Acknowledgements}

This work was carried out with the financial support from the Defence Research and Development Organization (Research and Training Scheme). We thank Prof. J Ramakrishna and Prof. N Kumar for encouraging us to carry out these investigations.

\section{References}

Delfyett P J, Dorsinville R and Alfano R R 1987 Opt. Lett. 121002

Dorsinville R, Delfyett P and Alfano R R 1987 (Weinhein/Bergstr: Verlag Chemie GmbH) Appl. Optics 263655

Fisher R A (ed.) 1983 Optical phase conjugation (New York: Academic Press)

Hellwarth R W 1977 Prog. Quantum Electron. 51

McCallum D S and Hutchinson M H R 1987 Opt. Commun. 64403

McCallun D S, Hutchinson M H R and Adrain R S 1988 Phys. Rev. A38 222

Pepper D M 1982 Opt. Eng. 21158

Saha S K and Hellwarth R W 1983 Phys. Rev. A27 919

Schrader B and Meier W 1974 Raman/IR atlas of organic compounds (Weinhein/Bergstr: Verlag Chemie $\mathrm{GmbH)}$ vols $1 \& 2$

Zel'dovich B Ya, Pilipetesky N F and Shkunov V V 1985 Principles of phase conjugation (Berlin: Springer Verlag) 